\title{
Exploiting Object Hierarchy: Combining Models from Different Category Levels
}

\author{
Alon Zweig Daphna Weinshall \\ School of Computer Science and Engineering \\ Hebrew university of Jerusalem, Israel 91904 \\ \{zweiga, daphna\}ecs.huji.ac.il
}

\begin{abstract}
We investigated the computational properties of natural object hierarchy in the context of constellation object class models, and its utility for object class recognition. We first observed an interesting computational property of the $o b$ ject hierarchy: comparing the recognition rate when using models of objects at different levels, the higher more inclusive levels (e.g., Closed-Frame Vehicles or Vehicles) exhibit higher recall but lower precision when compared with the class specific level (e.g., bus). These inherent differences suggest that combining object classifiers from different hierarchical levels into a single classifier may improve classification, as it appears like these models capture different aspects of the object. We describe a method to combine these classifiers, and analyze the conditions under which improvement can be guaranteed. When given a small sample of a new object class, we describe a method to transfer knowledge across the tree hierarchy, between related objects. Finally, we describe extensive experiments using $o b-$ ject hierarchies obtained from publicly available datasets, and show that the combined classifiers significantly improve recognition results.
\end{abstract}

\section{Introduction}

Human cognition relies on a hierarchal representation of objects in the world (see examples in Fig. 1), in the process of recognizing and referring to objects. How can we use such hierarchical structure to improve object recognition and categorization? This question has been addressed in a number of recent papers, mostly pursuing different directions to exploit this hierarchy when confronted with new categories (the small sample problem). The directions under study included the transfer of knowledge from known to new categories using Bayesian priors [11], sharing parts between objects at different levels of the hierarchy and improving generalization $[13,3]$, learning distance functions using related classes [6,9], and transferring features [10,4] or structure [5] from known classes to new ones. Often,

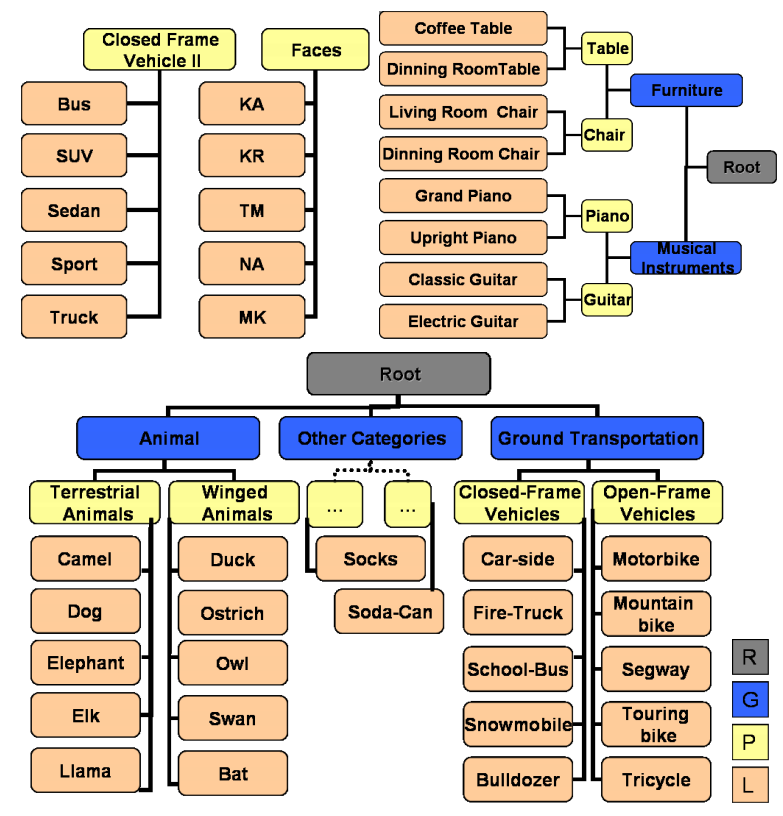

Figure 1. The four hierarchies used in our experiments. Categories at different levels of the tree are labeled (and color-coded) as follows: 'L' denotes the Leaf level, 'P' denotes the Parent level, 'G' denotes the Grandparent level, and ' $R$ ' the level of all objects. Our largest hierarchy (lowest diagram above) contains object classes from the CalTech256 database [8]. The facial hierarchy contains objects from [12].

when working on object class recognition, objects are represented by parts (or features) learned directly from example images of each object category, where relations between the parts (geometrical and possibly other) may sometimes be captured by graphical models trained using the same data.

We address the question posed above from a somewhat different point of view. We observe that the natural object hierarchy offers at our disposal a rich family of classifiers, for each category in each node of the hierarchy tree; the similarity between these classifiers varies, possibly in some relation to the distance between them on the object tree, but they all share some common characteristics. For example, if we build these classifiers with a discriminative algorithm that uses the background images of the CalTech256 
database [8], then all the classifiers are trained to distinguish a certain isolated object from a background of clutter. Such commonalities may permit the combination of different classifiers to improve the recognition of specific object classes. We expect this improvement to be more pronounced when using related objects, and in particular objects from higher (inclusive) levels in the hierarchy tree.

The idea of combining classifiers has been extensively studied in the machine learning community under different frameworks, including committee machines, ensemble average, or boosting. In light of the so-called bias/variance dilemma [7], the ensemble average of a number of classifiers should improve generalization, as long as the classifiers are both accurate and diverse. One common way to obtain such a collection of classifiers is to train different classifiers with different samples of the training data. But note that the object recognition classifiers, trained to recognize different objects at different levels of the object hierarchy tree, may be viewed as just such - classifiers trained on different resamples of the training data. Viewed this way, we may expect to see improvement when combining any set of classifiers, even those trained to recognize very distinct objects. At the same time, for obvious reasons, we expect to see larger improvement when combining classifiers trained to recognize similar objects (those closer to each other on the tree), as compared to very different ones.

Thus the main conceptual contribution of this paper is to identify the object hierarchy as a source of classifier variability, which is induced by different and inherently meaningful resampling of the training data. We then go ahead and describe a framework to combine the classifiers linearly, using each classifier's probabilistic output and the corresponding LRT (Loglikelihood Ratio Test) value. This approach is somewhat different, and possibly more powerful, than the traditional ensemble of classifiers, since each object classifier builds a different representation of the data based on the training subset that it sees. The approach differs from boosting and bagging in that the data resampling is not based on some bootstrapping procedure, but on supervised information given to the system via the object hierarchy tree. Clearly our approach could be augmented with boosting to further improve results.

The rest of this paper is organized as follows. First, we review in Section 2 the object class constellation model used to obtain each object classifier, and describe how we combine these classifiers. We also discuss the theory underlying our approach. In Section 4 we show that a number of intuitive object hierarchies (described in Section 3), provided by a human teacher, reveal consistent and sensible computational characteristics. Specifically, classifiers built for more specific objects (such as 'bus') - corresponding to the lowest level in the object tree, are characterized by high precision (or high specificity) and low recall (low sensitivity), while classifiers built for more general classes of objects at the next levels up the tree (such as 'vehicle') show the opposite - low specificity and high sensitivity. In general, the specificity of object classifiers decreases as we ascend the object tree. Then, In Section 5, we describe how to use the hierarchy to transfer knowledge between classes, as a way to address the small sample problem. Finally, we investigate in Section 6 combined classifiers as a general framework for the construction of object recognizers. We show empirically that combined classifiers improve performance significantly (over all constituent classifiers), and that typically three-level combinations perform better still than two-level combinations.

\section{Combining Object Models}

Our object class model To learn object models, we use the method described in [2], because its computational efficiency allows us to consider models (and images) with many features. The algorithm learns a generative relational part-based object model, modeling appearance, location and scale. Location and scale are relative to the unknown object location and scale, as captured by a star-like Bayesian network. The model's parameters are discriminatively optimized using an extended boosting process. This model has been shown to achieve competitive recognition results on standard benchmark datasets, approaching the state-of-the-art in object class recognition. Thus we believe that the results and improvements we show are general, and can be replicated with other, conceptually similar, part-based models.

Based on this model and some simplifying assumptions, the likelihood ratio test function is approximated (using the MAP interpretation of the model) by

$$
F(\mathbf{x})=\max _{C} \sum_{k=1}^{P} \max _{u \in Q(\mathbf{x})} \log p\left(u \mid C, \theta^{k}\right)-\nu
$$

with $P$ parts, threshold $\nu, C$ denoting the object's location and scale, and $Q(\mathbf{x})$ the set of extracted image features.

Classifier combination rule In our experiments we combined 2, 3 and 4 object classifiers. For each classifier, we used its LRT value from (1), obtaining a 2-, 3- or 4dimensional vector respectively. We then trained a Support Vector Machine classifier with the same training data represented in this new vector space, using a linear kernel.

The bias/variance dilemma Let $\mathrm{x} \in \mathcal{X}$ denote the image, $F(\mathbf{x})$ the LRT output of the classifier from (1), and $D(\mathbf{x})$ denote the binary random variable assigning 1 to class images, and -1 to background images. It can be readily 
shown that the mean-square error between classifier $F$ and the desired output $D$ can be decomposed as follows:

$$
\begin{aligned}
E\left[(F(\mathbf{x})-E[D(\mathbf{x})])^{2}\right] & =B(F(\mathbf{x}))+V(F(\mathbf{x})) \\
B(F(\mathbf{x})) & =(E[F(\mathbf{x})]-E[D(\mathbf{x})])^{2} \\
V(F(\mathbf{x})) & =E\left[(F(\mathbf{x})-E[F(\mathbf{x})])^{2}\right]
\end{aligned}
$$

where $B(F(\mathbf{x}))$ denotes the classifier's bias, and $V(F(\mathbf{x}))$ its variance. It can also be shown that when considering an ensemble average of such classifiers, the bias of the new classifier remains the same as the bias of $F(\mathbf{x})$, but its variance is reduced. As a result, the mean square error of the new classifier is also reduced.

The sensitivity/specificity tradeoff We now analyze the case of two classifier combination, where one classifier has high sensitivity and low specificity and the other has low sensitivity and high specificity. Recall that this is the computational property that distinguishes object classifiers from lower levels of the object hierarchy tree and classifiers from higher levels of the tree (see Section 4), and thus this analysis is revealing.

Given the function $F(\mathbf{x})$ from (1), define the classifier $F^{*}(\mathbf{x})=\operatorname{sign}(F(\mathbf{x}))^{1}$. Let $F_{1}(\mathbf{x}), F_{2}(\mathbf{x})$ denote two classification functions, and $G(\mathbf{x})=\frac{F_{1}(\mathbf{x})+F_{2}(\mathbf{x})}{2}$ its ensemble average. Let $G^{*}(\mathbf{x})=\operatorname{sign}(G(\mathbf{x}))$ denote the corresponding classifier, and let $G^{* *}(\mathbf{x})=\frac{F_{1}^{*}(\mathbf{x})+F_{2}^{*}(\mathbf{x})}{2}$ denote another related classifier.

We compute the error probability $P_{E}$ of classifier $G^{*}(\mathbf{x})$ :

$$
\begin{aligned}
& 4 P_{E}\left(G^{*}(\mathbf{x})\right)=E\left[\left(G^{*}(\mathbf{x})-D(\mathbf{x})\right)^{2}\right] \\
& =E\left[\left(\left(G^{*}(\mathbf{x})-G^{* *}(\mathbf{x})\right)+\left(G^{* *}(\mathbf{x})-D(\mathbf{x})\right)\right)^{2}\right] \\
& =E\left[\left(G^{*}-G^{* *}\right)^{2}\right]+E\left[\left(\frac{1}{2}\left(F_{1}^{*}-D\right)\right)^{2}\right] \\
& +E\left[\left(\frac{1}{2}\left(F_{2}^{*}-D\right)\right)^{2}\right]+2 E\left[\left(\frac{1}{2}\left(F_{1}^{*}-D\right)\right)\left(\frac{1}{2}\left(F_{2}^{*}-D\right)\right)\right] \\
& +2 E\left[\left(G^{*}-G^{* *}\right)\right]\left\{E\left[\frac{1}{2}\left(F_{1}^{*}-D\right)\right]+E\left[\frac{1}{2}\left(F_{2}^{*}-D\right)\right]\right\}
\end{aligned}
$$

Note that

$$
E\left[\left(\frac{1}{2}\left(F_{i}^{*}(\mathbf{x})-D(\mathbf{x})\right)\right)^{2}\right]=P_{E}\left(F_{i}^{*}\right)
$$

and

$$
\begin{array}{r}
E\left[\frac{1}{2}\left(F_{i}^{*}(\mathbf{x})-D(\mathbf{x})\right)\right]=P\left[F_{i}^{*}(\mathbf{x})=1, D(\mathbf{x})=-1\right] \\
-P\left[F_{i}^{*}(\mathbf{x})=-1, D(\mathbf{x})=1\right]=\Delta S\left(F_{i}^{*}\right)
\end{array}
$$

where $\Delta S\left(F_{i}^{*}\right)$ denotes the classifier's preference to either recall (sensitivity) or precision (a measure typically similar to specificity $)^{2}$, i.e., its sensitivity minus its specificity.

\footnotetext{
${ }^{1}$ For convenience, we define the sign function as $\operatorname{sign}(F)=1$ if $F \geq$ 0 , and $\operatorname{sign}(F)=-1$ if $F<0$.

${ }^{2}$ Notation reminder: recall and sensitivity denote the rate of true positives, specificity denotes the rate of true negatives, and precision denotes the fraction of true positives among all examples identified as positive.
}

Henceforth we shall call $\Delta S\left(F_{i}^{*}\right)$ the 'recall/precision primacy'. Finally,

$$
\begin{aligned}
E\left[\left(G^{*}-G^{* *}\right)^{2}\right] & =P\left(F_{1}^{*}=1, F_{2}^{*}=-1\right)+P\left(F_{1}^{*}=-1, F_{2}^{*}=1\right) \\
& \leq P_{E}\left(F_{1}^{*}\right)+P_{E}\left(F_{2}^{*}\right)
\end{aligned}
$$

(with equality only when $F_{1}^{*}, F_{2}^{*}$ err on disjoint sets of examples).

Putting all the above together, we get

$$
\begin{aligned}
4 P_{E}\left(G^{*}\right) \leq & \left.2 P_{E}\left(F_{1}^{*}\right)+2 P_{E}\left(F_{2}^{*}\right)+2 \Delta S\left(F_{1}^{*}\right) \Delta S\left(F_{2}^{*}\right)\right] \\
& +2 E\left[\left(G^{*}-G^{* *}\right)\right]\left(\Delta S\left(F_{1}^{*}\right)+\Delta S\left(F_{2}^{*}\right)\right) \\
& -2 \rho\left(\frac{1}{2}\left(F_{1}^{*}-D\right), \frac{1}{2}\left(F_{2}^{*}-D\right)\right)
\end{aligned}
$$

where $\rho(X, Y)=E[X Y]-E[X] E[Y]$ denotes the nonnormalized correlation coefficient of $X, Y$.

We can now state our main result:

Result: Assume that $F_{1}^{*}, F_{2}^{*}$ are two classifiers with opposite recall/precision primacy, i.e. and w.l.o.g., $\Delta S\left(F_{1}^{*}\right) \geq$ 0 and $\Delta S\left(F_{2}^{*}\right) \leq 0$; thus $\Delta S\left(F_{1}^{*}\right) \cdot \Delta S\left(F_{2}^{*}\right) \leq 0$. Assume further that the magnitude of their primacy is similar, i.e., $\left|\Delta S\left(F_{1}^{*}\right)\right| \approx\left|\Delta S\left(F_{2}^{*}\right)\right|$, and that their correlation with respect to the data is small, i.e., $\mid \rho\left(\frac{1}{2}\left(F_{1}^{*}-D\right),\left(\frac{1}{2}\left(F_{2}^{*}-\right.\right.\right.$ $D)))|<| E\left[\frac{1}{2}\left(F_{1}^{*}-D\right)\right] E\left[\frac{1}{2}\left(F_{2}^{*}-D\right)\right] \mid$. Then it follows from (3) that

$$
P_{E}\left(G^{*}\right) \leq \frac{P_{E}\left(F_{1}^{*}\right)+P_{E}\left(F_{2}^{*}\right)}{2}
$$

In other words, the error probability of the combined classifier is smaller (usually significantly so) than the mean error probability of the constituent classifiers.

In practice, we see that the combined error is typically smaller than the minimal error of the constituent classifiers with opposite recall/precision primacy, see Section 6.

\section{Datasets and General Experimental Setup}

\section{Datasets}

In our experiments, we used an extensive data set containing various objects that can be found in natural scenes. As much as possible, classes were taken from standard benchmark datasets, with a few exceptions (to be detailed shortly). We organized these objects into four natural hierarchies. Examples from the object classes and background images can be viewed in Fig. 2. A summary of the hierarchies is provided in Fig. 1.

In the rest of this paper we use the following notation to refer to object classes at different levels in the hierarchy (see Fig. 1): specific object classes, like 'Elk' and 'Tricycle', are labeled 'L' (for Leaf). More inclusive categories, like 'Terrestrial Animals', are labeled 'P' (for Parent). Categories at 


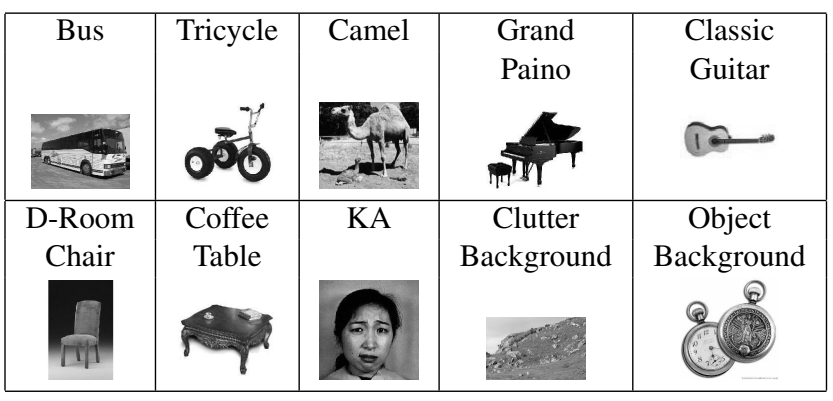

Figure 2. Examples taken from the object classes and background images, used to train and test our different Category level models, see Fig. 1.

the next level up, like 'Animals', are labeled 'G' (for Grandparent). Finally, the category of all objects is denoted ' $R$ ' (for Root).

For the discriminative learning procedure (see Section 2) and in order to evaluate the recognition results, we used two types of background. When using classes from the CalTech256 dataset [8] (the lowest hierarchy in Fig. 1), we used their Clutter Background images as well. With the remaining 3 smaller hierarchies and to achieve greater variability (and additional challenges) in the conditions of our experiments, we used our own Object Background dataset, containing various images of objects different from the learnt objects. This background was manually collected using Google, PicSearch and online catalogues.

CalTech256 Object Hierarchy This hierarchy includes pictures of various objects from the CalTech256 dataset [8], see Fig. 1. We chose objects that can be naturally organized into a sensible hierarchy: 'Animals' - including 'Terrestrial Animals' and 'Winged Animals', and 'Ground Transportation' - including 'Open-Frame Vehicles' and 'ClosedFrame Vehicles'. Models were learnt for objects from the 'Terrestrial Animals' and 'Open-Frame Vehicles' classes; other objects were used for training ' $G$ ' and ' $R$ ' level models. The CalTech256 Clutter Background was used with this dataset. We note that our category affiliations may not be identical to those used in [8], in an attempt to emphasize visual similarities over functional (thus ignoring, for example, the motorized vs. un-motorized distinction); we also used different names for the inclusive categories. The images in the Ground-Transportation Category were flipped to achieve uniform orientation (all vehicles pointing rightwards).

Closed-frame VehicleII Hierarchy This hierarchy contains 5 classes of common vehicles (more so than those in the CalTech256 database), contrasted with pictures from the 'Object Background'. Pictures (for the vehicle classes and background) were chosen manually from Google and PicSearch, showing vehicles at similar canonical orientation.
Faces Hierarchy This hierarchy contains pictures of 5 individuals taken from [12], with varying facial expressions.

Basic-Level Hierarchy This hierarchy was built to match standard hierarchies favored in the cognitive science literature, representing canonical categorization levels (basiclevel, sub-ordinate, and super-ordinate). Pictures were obtained from the CalTech101 subset of [8], or collected using mainly online shopping catalogues.

\section{General experimental setup}

In general, we always tested recognition performance for specific object categories from level 'L' (leaf) of all the respective hierarchies. Thus, for example, when comparing three models such as 'Llama', 'Animal', and 'Terrestrial Animal', they were all tested on the recognition of Llama pictures.

For each hierarchy, all models were trained with the same background images but different object images. All tests were done with the same background and test images, and the same algorithm parameters. Each experiment was repeated 60-100 times, with new random samples of train and test images. Since the Equal Error Rate (denoted EER) of the ROC is not well suited when the number of positive examples is much smaller than the number of negative examples [1], we used the preferred EER of the Recall Precision Curve (RPC).

To compare performance, we report two measures: (i) Precision and Recall of each classifier; (ii) EER of the RPC curve for each classifier, computed by varying the threshold of the optimal linear SVM classifier.

\section{Object Hierarchy}

We study the computational properties distinguishing objects from different levels in the object hierarchy tree, revealing opposite recall/precision primacy - high precision for the lowest level (specific) models, and high recall for higher level (inclusive) models. With sufficiently large samples per object, and given that we always test the recognition of object classes from level 'L', not surprisingly object models from level 'L' show superior recognition performance. In accordance, we see a decrease in performance as we use models ascending the object hierarchy tree.

Experimental setup We tested the recognition of each specific object from level 'L' by 5 types of models learnt using object categories from different levels in the hierarchy tree, see Table 1 . To assure fair comparison, all models saw the same train images of the 'L' object they were tested on; an illustrative example of this procedure is shown in Table 1. In different experiments we varied the number of train images per 'L' object: 5, 10, 15, 20, 25 and 30. Three 
hierarchies were used: CalTech256 Object, Closed-frame VehicleII, and Faces.

\begin{tabular}{|c|c|c|c|c|c|c|}
\hline \multirow[t]{2}{*}{ Exp } & \multicolumn{5}{|c|}{ Category training set } & Example: \\
\hline & $\begin{array}{ll}\mathrm{L} \\
\mathrm{H}\end{array}$ & $P$ & $\mathrm{G}$ & $\mathrm{R}$ & DB & Llama \\
\hline 1 & 1 & & & & & Llama \\
\hline 2 & & 5 & & & & $\begin{array}{l}\text { Llama, Camel, Dog, Elk } \\
\text { Elephant }\end{array}$ \\
\hline 3 & & & 5 & & & $\begin{array}{l}\text { Llama, Duck, Owl } \\
\text { Swan, Ostrich }\end{array}$ \\
\hline 4 & & & & 5 & & $\begin{array}{l}\text { Llama, Soda-can, Sock } \\
\text { Segway, Motorbike }\end{array}$ \\
\hline 5 & & & & & 5 & $\begin{array}{l}\text { Llama, Segway, Tricycle } \\
\text { Motorbike, Mountainbike }\end{array}$ \\
\hline
\end{tabular}

Table 1. This table shows the 5 different models learnt and evaluated on the recognition of ' $L$ ' level objects. 'DB' refers to a ' $G$ '-level category from a Different Branch of the tree. Examples are shown for the Llama as 'L' level object. In each different experiment, each 'L' class provided the same fixed amount of pictures to the training set $(5,10,15,20,25$ or 30 .)

With all the 3 hierarchies, we used test data composed of images of the target object and images of the relevant background in equal proportion. None of the test images was used for training. With the CalTech256 Object Hierarchy, where the models are learnt using the Clutter Background, we also conducted additional experiments, using for test data images from the target ' $L$ ' object mixed with images of a different ' $L$ ' object.

\subsection{Results}

Recall and Precision are shown in Fig. 3, comparing recognition when using ' $L$ ' and ' $P$ ' level models (left), and 'L' and ' $G$ ' level models (right). In the first comparison (Leaf vs. Parent), only 4 representative examples from the 3 hierarchies are shown; very similar results were obtained with all other objects. In the second comparison (Leaf vs. Grandparent), all objects from the CalTech256 Object Hierarchy are shown. These graphs clearly show the Recall/Precision Primacy effect, where 'L' models show high precision and low recall in recognition, while ' $P$ ' and ' $G$ ' models show high recall and low precision. This happens for all objects, regardless of the number of training examples.

The Recall Precision Curve and its corresponding EER are shown in Fig. 4 for two representative examples. Not surprisingly, we see that with sufficient training examples per 'L' object (as for the Dog class), the 'L' model performs best, and performance deteriorates as we ascend the object hierarchy. As the sample per class decreases, the advantage of the ' $L$ ' model over the ' $P$ ' model decreases, eventually the 'P' model might outperform the ' $\mathrm{L}$ ' model. Once again, similar phenomenon is observed for all objects.

Looking more closely at these results, we see that the
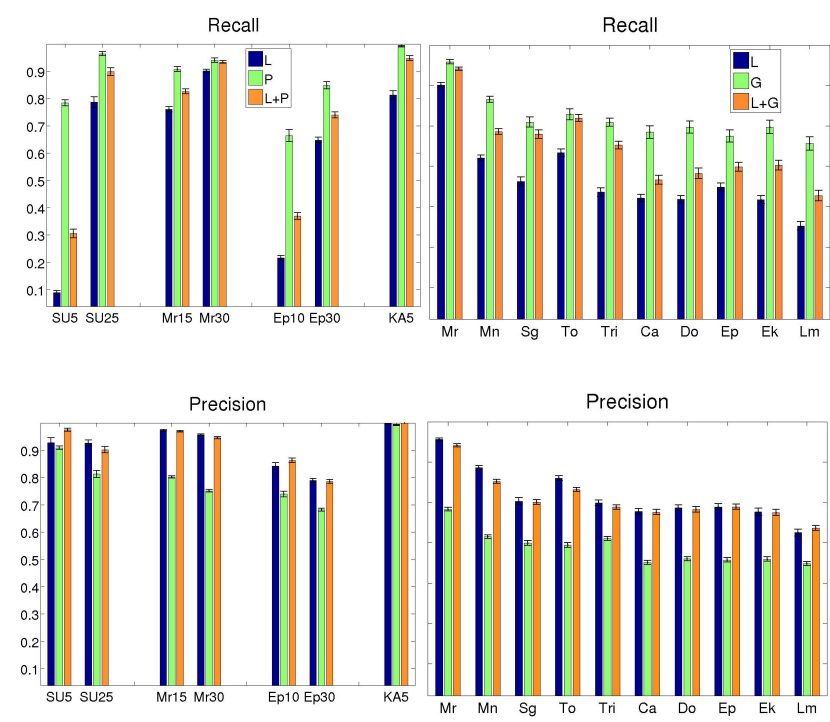

Figure 3. Recall and Precision of classifiers. Left column: four representative object classes, recognized with the ' $\mathrm{L}$ ', ' $\mathrm{P}$ ' and the combined ' $\mathrm{L}+\mathrm{P}$ ' models. 'SU' refers to the SUV class, 'Mr' - Motorbike, 'Ep' - Elephant, 'KA'- one of the female faces. The numbers indicate the size of the train set (e.g., 'SU5' refers to the SUV model trained with 5 SUV examples) Right column: all learnt object classes from the CalTech256 Object Hierarchy trained with 30 images per object class, and recognized with the ' $L$ ', ' $G$ ' and the combined ' $L+G$ ' models.

recall/precision primacy difference occurs regardless of the overall recognition rate. Specifically, for the Elephant with 10 training examples, we see from Fig. 4 that the 'P' model performs better than the ' $L$ ' model, and vice versa for the Elephant with 30 training examples. Still, Fig. 3 shows the same Recall/Precision primacy in both cases.

\section{Using hierarchy to transfer knowledge}

We study here how to transfer information between related objects, located nearby in the object hierarchy tree, to handle the problem of small sample or the appearance of new objects.

Experimental setup We tested the recognition of each specific object from level 'L' by 7 types of models learnt using object categories from different (more inclusive) levels in the hierarchy tree, see Table 2, which also shows an illustrative example of this procedure. The test background set consisted of 75 background images, while the test object set consisted of 30 images. Only the Basic-Level Hierarchy was used.

\subsection{Results}

Fig. 5 shows the results. Clearly the 'P' class model transfers information most effectively (seen in the superi- 

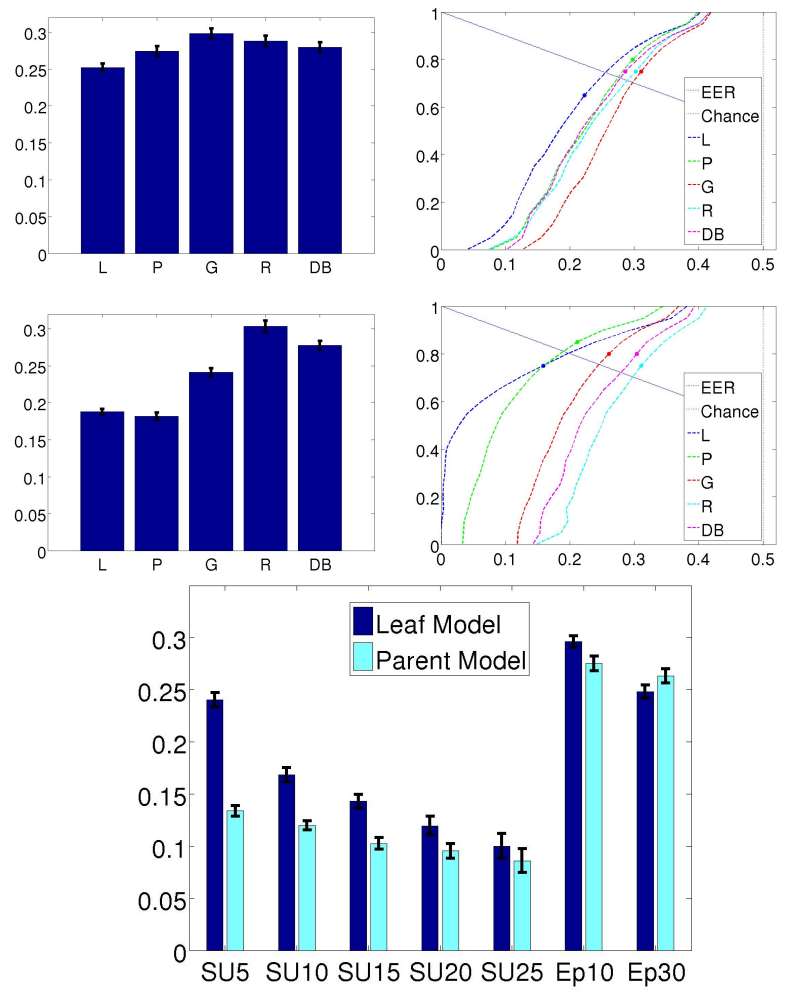

Figure 4. Performance of the different category level models. Top \& middle: left column - the EER of the RPC, right column - full RPC curve where the point of the original classifier is highlighted. Once again, 'L' denotes the Leaf category, 'P' - Parent, 'G' - GrandParent, 'R' - Root, and 'DB' - Different Branch. Top: performance of all models tested on the 'Dog' class from the Caltech256 hierarchy. Middle: same for the 'MountainBike' class from the Caltech256 hierarchy. Bottom: performance of the 'L' and 'P models on the 'SUV' class from the 'Closed-Frame VehiclesII' hierarchy and on the Elephant class from the 'Terrestrial Animals'. EER scores are shown as a function of the size of the training set - increasing from 5 up to 25 for the SUV, sizes 10 and 30 for the Elephant. Note the decrease in the performance superiority of the SUV ' $\mathrm{P}$ ' model over the 'L' model till it is insignificant, as the train size increases. Note the opposite superiority of 'L' vs. 'P' models when comparing the two Elephant class models.

ority of Exp. 3 over Exp. 5-7), and improves performance over the small sample case (seen in the superiority of Exp. 4 over Exp. 2).

\subsection{Discussion}

The results above show a clear hierarchy structure, where models which are learnt from nearby objects (brothers) in the object hierarchy tree can substantially improve the recognition results of each other. It shows the possibility for the success of a learning-to-learn scheme - where fewer examples of the goal object class are used in the learning process, augmented by examples from different related classes.

\begin{tabular}{|c|c|c|c|c|c|c|}
\hline \multirow[t]{2}{*}{ Ex } & \multicolumn{5}{|c|}{ Object training set } & \multirow{2}{*}{$\begin{array}{l}\text { Example: } \\
\text { Classic Guitar }\end{array}$} \\
\hline & $\mathrm{L}$ & $\mathrm{P}$ & $\mathrm{G}$ & $\mathrm{DB}$ & BG & \\
\hline$\overline{11}$ & 35 & & & & & Classic Guitar \\
\hline 2 & 1 & & & & & Classic Guitar \\
\hline 3 & & 30 & & & & Electric Guitar \\
\hline 4 & 1 & 30 & & & & Classic, Electric Guitar \\
\hline 5 & & & 30 & & & Grand Piano \\
\hline 6 & & & & 30 & & Living Room Chair \\
\hline 7 & & & & & 30 & Background \\
\hline
\end{tabular}

Table 2. The models learnt in the different experiments on the transfer of information between classes. 'L' refers to the Leaf level, 'P' to the Parent, ' $G$ ' to the Grandparent, 'DB' to a Different Branch of the tree, and 'BG' to the background.

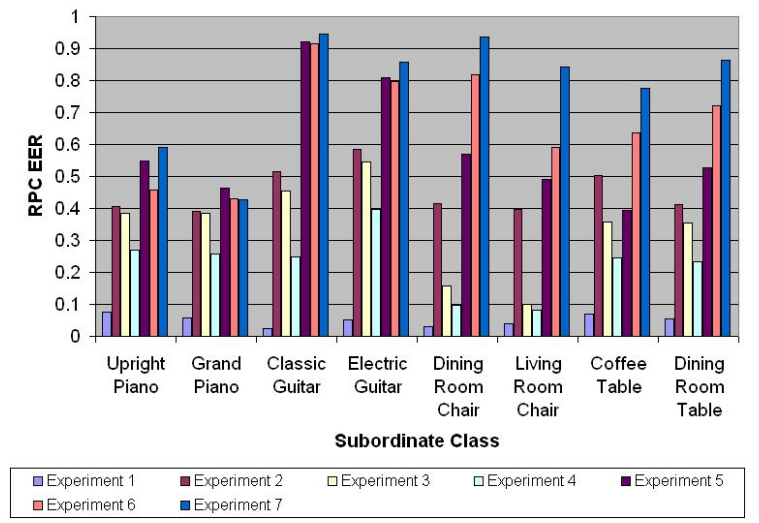

Figure 5. Transfer of knowledge between related classes. Description of the different experiments is given in Table 2 .

\section{Using hierarchy to improve classification via combination}

We now ask whether the combination of two or more object model classifiers, which are based on different category levels, can improve the performance of the original classifiers.

Experimental setup We used the same setup, same data, and same learnt models as used in Section 4. We tested different combinations by combining two or more models from different category levels as described in Section 2. The different combinations that we studied are summarized in Table 3.

For comparison, we used a naïve alternative method, which learned directly an object model using the same set of images as used by the combined model. Each image in this set was initially weighted to reflect its true weight on the combined model. For example, when combining two models such as 'Llama' and 'Terrestrial Animals', we note that the Llama images provided all the training set for the Llama model, and only $20 \%$ of the training set for the 'Ter- 


\begin{tabular}{|l|l|}
\hline Exp & $\begin{array}{l}\text { Example: } \\
\text { Llama as Leaf Level }\end{array}$ \\
\hline \hline L+P & 'Llama' + 'Terrestrial Animals' \\
\hline L+G & 'Llama' + 'Animals' \\
\hline L+R & 'Llama' + 'Tree Root' \\
\hline L+DB & 'Llama' + 'Open-Frame Vehicles' \\
\hline P+G & 'Terrestrial Animals' + 'Animals' \\
\hline L+P+G & 'Llama' + 'Terrestrial Animals' + 'Animals' \\
\hline L+P+G+R & $\begin{array}{l}\text { 'Llama' + 'Terrestrial Animals' + 'Animals' } \\
\text { +'Tree Root' }\end{array}$ \\
\hline
\end{tabular}

Table 3. The different combinations we studied: ' + ' denotes a combination of two models. 'L' refers to the Leaf level, 'P' - Parent, 'G' - Grandparent, 'R' - Root, and 'DB' - Different Branch.
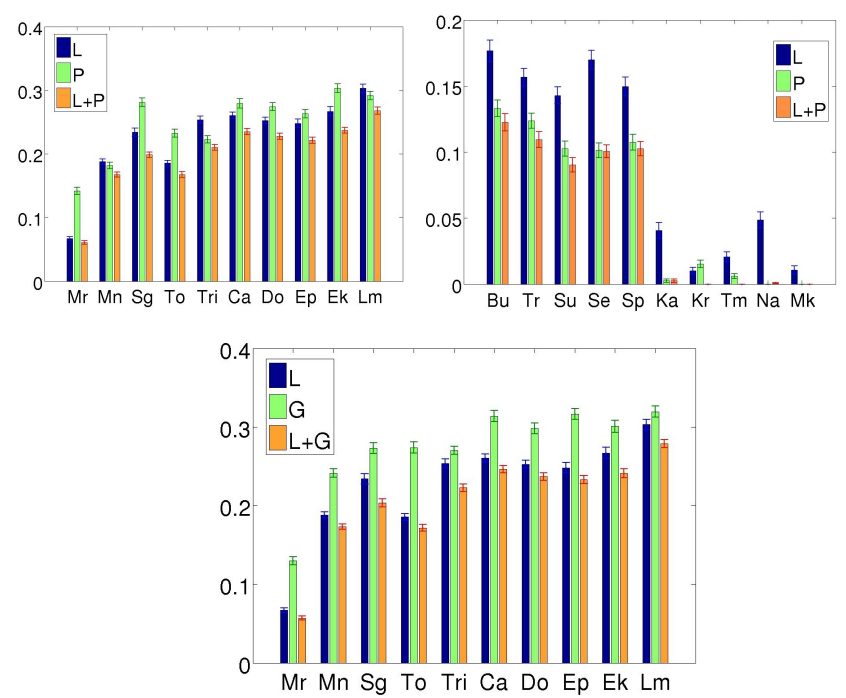

Figure 6. Comparison of combined and original classifiers. ' $L$ ' denotes a leaf model, 'P' - parent model, 'G' - grandparent model, 'L+P' leaf/parent combined model and ' $\mathrm{L}+\mathrm{G}$ ' leaf/grandparent combined model. Top: object models recognized with the ' $\mathrm{L}$ ', 'P' and the combined ' $\mathrm{L}+\mathrm{P}$ ' models. Bottom: object models recognized with the ' $\mathrm{L}$ ', 'G' and the combined 'L+G' models. Top-Left: CalTech256 Object Hierarchy, 'Open-frame Vehicle' and 'Terrestrial Animal', with 30 training images per object class. Specifically, 'Mr' denotes the Motorbike class, 'Mn' - Mountain-Bike , 'Sg' - Segway, 'To' - Touring-Bike, 'Tri' - Tricycle, 'Ca' - Camel, 'Do' Dog, 'Ep' - Elephant, 'Ek' - Elk, 'Lm' - Llama. Top-Right: 'Closed-Frame VehicleII' Hierarchy with 15 training images per object class, and 'Faces' Hierarchy with 5 training images per object class. Bottom: CalTech256 Object Hierarchies, with 30 training images per object class

restrial Animals' model; thus in the training of the combined model, the Llama training set received total weight of 0.6 , while the remaining 4 classes received total weight of 0.4 . The background train set remained unchanged.

\subsection{Results}

Fig. 6 shows the EER recognition results for all 20 objects, from the 'P' classes of 'Terrestrial Animals', 'Open-
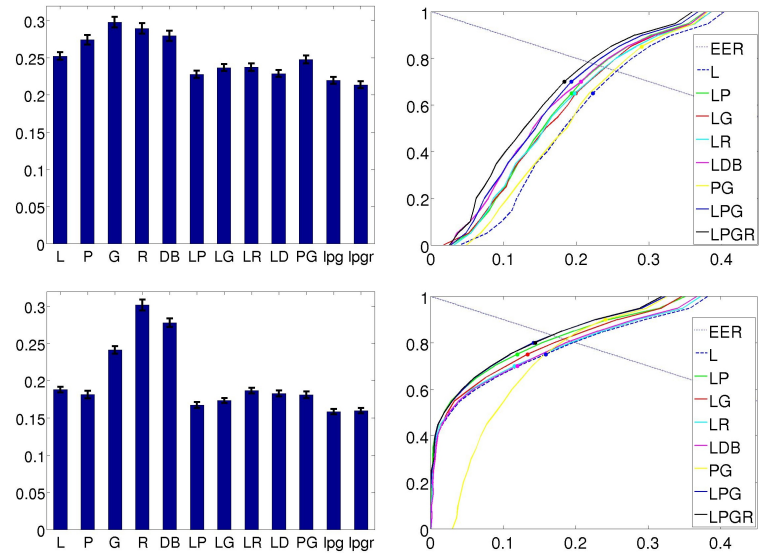

Figure 7. All different combinations of classifiers. Left: the EER of the RPC. Right: full RPC (Recall Precision Curves). Top: results when recognizing the 'Dogs' class. Bottom: results with the 'Mountain Bike' class.

Frame Vehicles', 'Closed-Frame VehiclesII', and 'Faces'. We show recognition results with 3 models - 'L', 'P', and the combined ' $\mathrm{L}+\mathrm{P}$ ', fixing the number of training examples to $30,30,15$, and 5 for each object in the 4 ' $\mathrm{P}$ ' classes respectively. We also show recognition results with 3 models - 'L', 'G', and the combined 'L+P', with 30 training examples and two classes of the CalTech256 Object Hierarchy.

Clearly, almost always, the combined model performed better than both constituent models. This happened for all objects and all training conditions, regardless of which of the constituent models was initially superior. The only exception occurred in the experiments with only 5 training images per object class (small sample). Moreover, in all experiments the combined model improved significantly the weak measure (either Recall or Precision) of each of the constituent models, as demonstrated in Fig. 3.

Fig. 7 shows results with the 7 different classifier combinations, listed in Table 3, for two object classes. These are representative results - similar results were obtained with all other classes. Note that the two-level combinations that obtain the highest performance are either the ' $\mathrm{L}+\mathrm{P}$ ' or ' $\mathrm{L}+\mathrm{G}$ '. Not surprisingly, therefore, the best results are obtained with the three- and four-level combinations $(' \mathrm{~L}+\mathrm{P}+\mathrm{G}$ ' and ' $\mathrm{L}+\mathrm{P}+\mathrm{G}+\mathrm{R}$ ' respectively).

Fig. 8 shows the EER of the RPC in the second test condition, when test examples included an equal number of images from the target object and another unrelated distractor object (instead of the standard background images). Not surprisingly, when the 'L' model was combined with a model whose training set included pictures of the distractor object, the performance of the combined model was reduced. However, interestingly enough, this reduction is rather slight (see Fig. 8). This decrease remains slight even when the ' $L$ ' model is combined with a very poor classifier (under these conditions), like the 'R' or 'DB' ones. Thus 

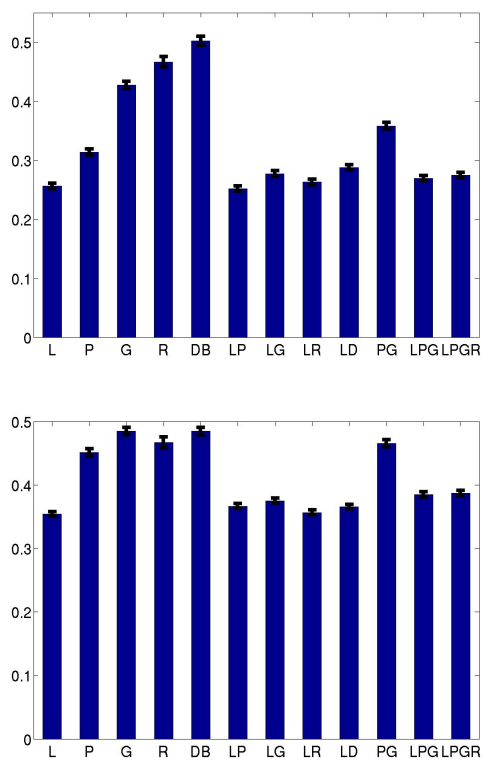

Figure 8 . The EER of the RPC when the test examples included images of the target object - the 'Mountain Bikes' - and another object (instead of the standard background images). Top: models tested against 'Camel'. Bottom: models tested against 'Tricycle'.

these results seem to suggest that the improvement obtained by the combined classifier against the standard background is not accomplished at the high cost of reducing the discriminability of the new classifier against other, possibly related, objects.

Finally, we note that in most cases, the comparison against the naïve model showed superior results for the combined model, while in the other cases the advantage of the naïve model was not significant. On the other hand, the training time of the naïve model was substantially longer. Moreover, this training procedure is not modular, while the combination scheme we described is rather flexible.

\section{Summary and Discussion}

We analyzed the computational properties of constellation object class models, built to describe object categories at different levels of the object hierarchy tree. An interesting observation emerged, when comparing specific object models, trained using images of objects corresponding to the leaves of the hierarchy tree, with models built to describe categories at higher levels of the object hierarchy tree. The first (specific) models exhibit higher precision, while the second (inclusive) models exhibit higher recall. We provided the theoretical analysis showing why this situation should be favorable for the success of a classifier combined from two such constituents (one with higher precision, the other with higher recall), and demonstrated experimentally that significant improvement is indeed achieved in all cases. In our experiments the combined model performed better than all constituents models in almost all cases. The improvement magnitude was larger when the constituent classifiers corresponded to nearby objects in the hierarchy tree, showing that this improvement is not due simply to the larger training set.

In all our experiments, we used a specific part-based model that can be learned rather efficiently, and can therefore handle a relatively large number of parts (or features). Although we did not perform experiments to this effect, we believe that this improvement can be obtained with any object class model, and that the phenomena we have observed do not depend on the specific model we used.

\section{References}

[1] S. Agarwal and D. Roth. Learning a sparse representation for object detection. In Proc. ECCV, 2002. 4

[2] A. Bar-Hillel, T. Hertz, and D. Weinshall. Efficient learning of relational object class models. Proc. ICCV, 2005. 2

[3] A. Bar-Hillel and D. Weinshall. Subordinate class recognition using relational object models. Proc. NIPS, 19, 2006. 1

[4] E. Bart and S. Ullman. Cross-generalization: learning novel classes from a single example by feature replacement. Proc. CVPR, pages 672-679, 2005. 1

[5] A. Ferencz, E. Learned-Miller, and J. Malik. Building a classification cascade for visual identification from one example. Proc. ICCV, pages 286-293, 2005. 1

[6] M. Fink. Object classification from a single example utilizing class relevance metrics. Proc. NIPS, 17, 2004. 1

[7] S. Geman, E. Bienenstock, and R. Doursat. Neural networks and the bias/variance dilemma. Neural Comput., 4(1):1-58, 1992. 2

[8] G. Griffin, A. Holub, and P. Perona. Caltech-256 object category dataset. Technical Report UCB/CSD-04-1366, California Institute of Technology, 2007. 1, 2, 4

[9] T. Hertz, A. Bar-Hillel, and D. Weinshall. Learning distance functions for image retrieval. Proc. CVPR, 2, 2004. 1

[10] K. Levi, M. Fink, and Y. Weiss. Learning From a Small Number of Training Examples by Exploiting Object Categories. LCVPRO4 workshop on Learning in Computer Vision, 2004. 1

[11] F. Li, R. Fergus, and P. Perona. One-shot learning of object categories. IEEE PAMI, 28(4):594-611, 2006. 1

[12] M. Lyons, S. Akamatsu, M. Kamachi, and J. Gyoba. Coding facial expressions with gabor wavelets. Proc. ICAFGR, pages 200-205, 1998. 1, 4

[13] E. Sudderth, A. Torralba, W. Freeman, and A. Willsky. Learning hierarchical models of scenes, objects, and parts. Proc. ICCV, 2005. 1 